\title{
GLUTAMINA E NUCLEOTÍDEOS NA DIETA DE FRANGOS DE CORTE CRIADOS NO SISTEMAALTERNATIVO
}

\author{
GLUTAMINE AND NUCLEOTIDE SUPPLEMENTATION IN BROILER DIETS IN \\ ALTERNATIVEBREEDING SYSTEM
}

\author{
Zavarize, K.C. ${ }^{1 *}$, Sartori, J.R. ${ }^{1 A}$, Pelícia, V.C. ${ }^{1 B}$, Pezzato, A.C. ${ }^{1 C}$, Araujo, P.C. ${ }^{1 D}$, \\ Stradiotti, A.C. ${ }^{1 \mathrm{E}}$ e Madeira, L.A. ${ }^{1 \mathrm{~F}}$
}

\begin{abstract}
${ }^{1}$ Faculdade de Medicina Veterinária e Zootecnia. Universidade Estadual Paulista. Botucatu, SP. Brasil. *kelen_zavarize@yahoo.com.br; Ajrsartori@fca.unesp.br; Bvcpelicia@yahoo.com.br; ccpezzato@fca.unesp.br; Dpricavalca@hotmail.com; Eanastradiotti@yahoo.com.br; Flucienemadeira@yahoo.com.br
\end{abstract}

\section{PALAVRAS CHAVE ADICIONAIS}

Desempenho. Morfologia intestinal.

\section{RESUMO}

Objetivou-se avaliar o efeito da suplementação com glutamina e nucleotídeos na dieta sobre o desempenho e morfologia da mucosa intestinal de frangos de corte criados no sistema alternativo. Foram utilizados 600 pintos de corte machos distribuídos em delineamento em blocos casualizados, no esquema fatorial $3 \times 2$ (três níveis de glutamina: 0,0; 0,5 e 1,0\% e dois níveis de nucleotídeos purificados na dieta: 0,0 e 0,04\%), totalizando 6 tratamentos, com 4 repetições de 25 aves cada. Foram obtidos os dados de desempenho (peso corporal, ganho de peso, consumo de ração, conversão alimentar e mortalidade) aos 7, 21 e 42 dias de idade. Ao final do experimento foram abatidas 4 aves/tratamento para avaliação do peso de órgãos e da morfologia intestinal. A adição de 1\% na dieta melhorou o ganho de peso, consumo de raçao e conversão alimentar na primeira semana de idade. Não sendo encontrados resultados positivos nos períodos de 21 e 42 dias de idade. A suplementação de glutamina e nucleotídeos em dietas de frangos de corte criados no sistema alternativo não influencia o desempenho e a morfologia intestinal, a inclusão de 1,0\% de glutamina no período de 1 a 21 dias de idade, favorece o desempenho das aves.

\section{SUMMARY}

The experiment was conducted to evaluate

\author{
AdDitionAL KEYWORDS \\ Performance. Intestinal morphology.
}

the effect of supplementing glutamine and nucleotides on growth performance and development intestinal morphology in broiler chicks. In the trial, 600 male broiler chicks distributed in randomized blocks in a $3 \times 2$ factorial arrangement (consisting of a uniform basal diet supplemented with: $0.0,0.5$ or $1.0 \%$ glutamine, and 0.0 or $0.04 \%$ nucleotides), for a total of 6 treatments with 25 birds each. Means of performance (weight, weight gain, feed intake, feed conversion and mortality) were obtained 7,21 and 42 days of age. At the end of the experiment 4 broilers per treatment were used to determine organs weight and development intestinal morphology. Glutamine supplementation (1\%) improved the body weight, feed intake and feed conversion in the first week. Glutamine and nucleotides supplementation did not affect performance in broiler chicks in the period one to 21 and one to 42 days of age. In conclusion, it was demonstrated that feeding 1.0\% glutamine improved growth performance of broiler birds at 21 days of age.

\section{INTRODUÇÃO}

No Brasil, como em outros países, temse observado crescente preocupação dos consumidores com a origem e qualidade dos produtos, exigindo alimentos saudáveis e ausência de resíduos. Com isso, as empre- 
sas avícolas estão se voltando para produção de frango em sistema alternativo à exploração industrial intensiva, produzindo aves criadas com rações sem antibióticos, anticoccidianos, promotores de crescimento, quimioterápicos e ingredientes de origem animal na sua composição, além de uma série de outros requisitos e normas aprovadas e oficializadas no âmbito da Associação da Avicultura Alternativa (Demattê Filho e Mendes, 2001).

O sistema alternativo da produção de frangos de corte tem como objetivo a obtenção de produtos com atributos diferenciados e de qualidade certificada, utilizando-se tecnologias adequadas, respeitando o bem-estar animal, a saúde do homem, o meio ambiente e oferecendo produtos com segurança alimentar, garantindo rastreabilidade, satisfação e confiança dos consumidores (Butolo, 2003).

Existem vários produtos que podem ser utilizados para substituir os antibióticos promotores de crescimento nas dietas de frangos de corte e poedeiras, tais como probióticos, prebióticos, enzimas, extratos vegetais, entre outros. Assim, a suplementação com glutamina e nucleotídeos na dieta para frangos de corte pode desempenhar papel importante durante períodos de grandes desafios e para animais criados no sistema alternativo de produção.

Estudos demonstram que os nucleotídeos dietéticos são importantes para tornar disponíveis bases nitrogenadas e nucleosídeos, que podem ser utilizados imediatamente na síntese de nucleotídeos pela via de salvamento. Essa via é extremamente importante para os tecidos e órgãos cuja síntese de nucleotídeos é deficiente, mas que apresentam uma rápida divisão mitótica, como cérebro, eritrócitos, medula óssea, mucosa intestinal e linfócitos. Pois, nestes tecidos há grande demanda de ácidos nucléicos para atender a rápida divisão mitótica. As células de rápido crescimento, como enterócitos, apresentam capacidade limitada para síntese de purinas e pirimidinas pela síntese de novo, portanto é necessária a suplementação exógena para manter o pool de nucleotídeos (Uauy et al., 1994).

A glutamina tem grande importância nos processos metabólicos celulares, sendo indispensável para o crescimento da maioria das células e tecidos (Pierzynowski et al., 2001). Sob condições de elevada degradação de proteína, a glutamina pode atuar como regulador metabólico para aumentar a síntese e reduzir o catabolismo protéico. Tais circunstâncias incluem períodos de estresse e períodos de crescimento rápido dos tecidos, em que a síntese endógena pode não ser suficiente (Lobley et al., 2001). É considerado o principal substrato energético para células de proliferação rápida, tais como enterócitos e linfócitos ativos (Dewitt, 1999).

A suplementação da ração de leitões com 1,0\% de glutamina preveniu a atrofia dos vilos da mucosa intestinal na primeira semana pós-desmame e melhorou o desempenho na segunda semana pósdesmame. Isto pode ser atribuído ao fato de a glutamina servir como substrato energético para os enterócitos, contribuindo assim para o fornecimento de energia necessária ao desempenho das atividades metabólicas normais desse grupo de células, melhorando a digestão e a absorção de nutrientes, pois o desmame é considerado uma fase crítica do desenvolvimento de leitões (Wu et al., 1996).

Dell'Orto et al. (2002) trabalhando com $0,5 \%$ nucleotídeos e $0,5 \%$ glutamina para suínos observaram aumento na altura das vilosidades e profundidade de cripta do íleo, mostrando o efeito positivo sobre o crescimento e maturação da mucosa ileal. Da mesma maneira, Yu et al. (2002) avaliando a suplementação de nucleotídeos e glutamina para leitões desmamados, observaram maior tamanho das vilosidades do duodeno e jejuno.

Calson et al. (2005) observaram melhora no desempenho suínos na fase de creche e 
terminação ao fornecerem extrato de levedura como fonte de nucleotídeos, e atribuíram isso à melhora na integridade do trato intestinal e, consequentemente, na digestão e absorção de nutrientes. Wu et al. (1996) e Tucci (2003), suplementando glutamina na dieta de suínos na fase de creche, observaram melhora no desempenho dos animais.

A pesquisa teve por objetivo avaliar o efeito da suplementação com glutamina e nucleotídeos na dieta sobre o desempenho e morfologia da mucosa intestinal de frangos de corte criados no sistema alternativo.

\section{MATERIALE MÉTODOS}

O experimento foi conduzido na Universidade Estadual Paulista. UNESP, Câmpus de Botucatu, no Laboratório de Nutrição de Aves da Faculdade de Medicina Veterinária e Zootecnia, no período de setembro a outubro de 2006. Foram utilizados 600 pintainhos de corte machos, da linhagem Hybro, com um dia de idade, vacinados no incubatório contra doenças de Gumboro, Marek e bouba aviária (avian diphtheria). Foram distribuídas 25 aves/ boxe, na densidade de 10 aves $/ \mathrm{m}^{2}$, conforme recomendação para o sistema alternativo de criação de frangos de corte (Demattê Filho e Mendes, 2001).

Aos três dias de idade, as aves foram vacinadas contra coccidiose (Paracox ${ }^{\circledR}-5$ da Schering Plough Coopers), via água de bebida, conforme recomendações do fabricante. Aos 8 e 14 dias de idade, foram vacinadas contra a doença de Gumboro, com a vacina viva liofilizada CEVAC GUMBOL ${ }^{\circledR}$ e CEVAC IBD L ${ }^{\circledR}$ da Ceva Santé Animale, respectivamente, seguindo-se o mesmo procedimento para a vacinação anterior.

O fornecimento de água e ração foi $a d$ libitum, utilizando bebedouro e comedouro inicial, substituídos gradativamente por bebedouro pendular e comedouro tubular definitivos. Temperatura e ventilação foram controladas manualmente, manejando as cortinas laterais do galpão. O programa de luz foi natural, sem uso de iluminação durante a noite, a partir do $7^{\circ}$ dia de idade, quando foram retiradas as campânulas.

As aves foram distribuídas em delineamento em blocos casualizados no esquema fatorial 3 x 2 (três níveis de glutamina na dieta: 0,$0 ; 0,5$ e $1,0 \%$ e dois níveis de nucleotídeos purificados na dieta: 0,0 e 0,04\%), com quatro repetições de 25 aves cada, totalizando 100 aves por tratamento.

O programa de arraçoamento foi dividido em quatro fases: pré-inicial: 1 a 7 dias, inicial: 8 a 21 dias, crescimento: 22 a 35 dias e final: 36 a 42 dias adaptados de Rostagno et al. (2005), com rações isoprotéicas e isoenergéticas, sendo que o amido de milho e casca de arroz foram substítuidos gradativamente pela glutamina e pelos nucletídeos, respectivamente (tabela I). $\mathrm{Na}$ composição das rações não foram utilizados ingredientes de origem animal, conforme recomendação para o sistema alternativo de criação de frangos de corte (Demattê Filho e Mendes, 2001).

Nos períodos acumulados de 1-7, 1-21 e 1-42 dias de idade, foram obtidas as seguintes variáveis de desempenho: peso corporal, ganho de peso, consumo total de ração, conversão alimentar, fator de produção e viabilidade.

Na obtenção das amostras para peso de órgãos e morfologia intestinal aos 42 dias de idade, foram calculadas as médias de peso dos boxes, sendo retirada uma ave, na média de peso por boxe, sacrificada por deslocamento da articulação crânio-cervical, totalizando quatro aves por tratamento. Foram colhidos fígado, pró-ventrículo, moela, pâncreas, intestino delgado e intestino grosso. O fígado foi pesado imediatamente após ter sido retirado. O pró-ventrículo e moela foram abertos e pesados após remoção do conteúdo. Os intestinos delgado e grosso foram separados por secções no local onde o duodeno emerge da moela e no início dos cecos, sendo posteriormente pesados e medidos. O pâncreas foi pesado 
após ser removido do intestino delgado.

Nas análises histológicas foram colhidos dois segmentos de $3 \mathrm{~cm}$ do duodeno, do jejuno e do íleo, cortados transversalmente e longitudinalmente, abertos pela borda mesentérica, lavados e estendidos pela túnica serosa, os quais foram fixados em solução de formalina a $10 \%$ por 24 horas. Posteriormente, foram lavados em água corrente e armazenados em álcool 70\%, e, em seguida, desidratados em uma série crescente de álcoois, diafanizados em xilol e incluídos em Paraplast Plus ${ }^{\circledR}$. Com o uso de um micrótomo, foram obtidos cortes de $7 \mathrm{~m}$ de espessura, os quais foram corados com ácido periódico de Schiff (PAS).

Posteriormente, com o auxílio de microscópio ótico acoplado a sistema analisador de imagens Leica (DMLB - Leica Qwin) e computador, foram feitas medidas de altura e área das vilosidades, profundidade das criptas e contagem de enterócitos e células

Tabela I. Composição química e valores calculados das rações de frangos de corte. (Chemical composition and calculated values for broiler diets).

\begin{tabular}{|c|c|c|c|c|}
\hline Alimentos/Tratamentos ${ }^{1}$ & Pré-inicial & Inicial & Crescimento & Final \\
\hline Milho moído & 54,760 & 57,993 & 61,042 & 65,120 \\
\hline Farelo de soja & 37,450 & 34,600 & 30,910 & 27,100 \\
\hline Óleo de soja & 2,106 & 2,300 & 3,201 & 3,165 \\
\hline Fosfato bicálcico & 1,950 & 1,805 & 1,665 & 1,520 \\
\hline Calcário calcítico & 0,910 & 0,880 & 0,840 & 0,790 \\
\hline DL-Metionina & 0,250 & 0,175 & 0,175 & 0,170 \\
\hline L-Lisina & 0,380 & 0,210 & 0,235 & 0,275 \\
\hline L-Treonina & 0,150 & 0,100 & 0,100 & 0,100 \\
\hline Suplemento vitamínico ${ }^{1}$ & 0,025 & 0,025 & 0,025 & 0,025 \\
\hline Suplemento mineral ${ }^{2}$ & 0,050 & 0,050 & 0,050 & 0,050 \\
\hline $\mathrm{Sal}(\mathrm{NaCl})$ & 0,520 & 0,500 & 0,450 & 0,400 \\
\hline Cloreto de colina $60 \%$ & 0,060 & 0,060 & 0,050 & 0,040 \\
\hline Casca de arroz & 0,389 & 0,302 & 0,257 & 0,245 \\
\hline Amido de milho & 1,000 & 1,000 & 1,000 & 1,000 \\
\hline AccelerAid $^{3}$ & 0,000 & 0,000 & 0,000 & 0,000 \\
\hline Glutamina & 0,000 & 0,000 & 0,000 & 0,000 \\
\hline Energia metabolizável (kcal/kg)4 & 2.950 & 3.000 & 3.100 & 3.150 \\
\hline Proteína bruta, $\%{ }^{4}$ & 22,05 & 20,81 & 19,41 & 18,05 \\
\hline Cálcio, $\%^{4}$ & 0,94 & 0,89 & 0,83 & 0,76 \\
\hline Fósforo disponível, $\%{ }^{4}$ & 0,47 & 0,44 & 0,41 & 0,38 \\
\hline Fibra bruta, $\%^{4}$ & 3,11 & 2,98 & 2,82 & 2,68 \\
\hline Metionina, $\%^{4}$ & 0,58 & 0,49 & 0,47 & 0,45 \\
\hline Lisina, $\%^{4}$ & 1,47 & 1,26 & 1,19 & 1,12 \\
\hline Treonina, $\%{ }^{4}$ & 0,99 & 0,90 & 0,84 & 0,79 \\
\hline Aminoácidos sulfurados,$\%^{4}$ & 0,92 & 0,82 & 0,79 & 0,75 \\
\hline
\end{tabular}

${ }^{1}$ Nutron Nutrição Animal. Suplemento vitamínico (por kg de ração): vit. A, 8.000 UI; vit. D3, 2.000 UI; vit. E, 15 mg; vit. K, 1,8 mg; vit. B1, 1,8 mg; vit. B2, 6 mg; vit. B6, 2,8 mg; vit. B12, 12 mcg; niacina, 40 mg; ác. pantotênico, $15 \mathrm{mg}$; biotina, 0,06 mg; ac. fólico, $1 \mathrm{mg}$; Selênio, 0,3 ppm; BHT, 0,002 g. ${ }^{2}$ Nutron Nutrição Animal. Suplemento mineral (por kg de ração): I, 0,75 ppm; Fe, 50 ppm; Cu, 8 ppm; Mn, 75 ppm; Zn, 70 ppm. ${ }^{3}$ AccelerAid Formil Vet - Bixina (Mín/Max): 0,72\% / 0,90\%; Dióxido de silício (Mín): 1,192\%; Nucleotídeos totais (Mín): 22,50\%; Óxido de alumínio (Mín): 8,5320\%. ${ }^{4}$ Valores calculados. 
caliciformes do duodeno, jejuno e íleo, para determinação da relação células caliciformes/enterócitos.

As análises estatísticas dos dados de desempenho, peso de órgãos e morfologia intestinal foram feitos pelo método de análise de variância (ANOVA), com o auxílio do procedimento General Linear Model do SAS (1996) e, quando necessário, foi utilizado o teste de Tukey ao nível de 5,0\% de probabilidade, para verificar diferenças significativas entre as médias dos tratamentos.

\section{RESULTADOSEDISCUSSÃO}

O ganho de peso e o consumo de ração das aves foram influenciados no período de 1 a 21 dias de idade com a inclusão de 1,0\% de glutamina na dieta $(\mathrm{p}<0,05)$, conforme mostrado na tabela II. Evidenciando a importância do aminoácido para a fase inicial do desenvolvimento animal, pois necessitam de maior quantidade de nutrientes (Calson et al., 2005; Maiorka et al., 2001; Wu et al., 1996).

As demais carcaterísticas de desempenho avaliadas nos demais períodos não foram influenciados com a inclusão de glutamina e nucleotídeos (tabela II). Isto se deve, provavelmente, ao fato das rações estarem balanceadas e à excelente qualidade dos ingredientes, além dos pintos terem

Tabela II. Peso inicial (PI), peso final (PF), ganho de peso médio (GP), consumo de ração $(\mathrm{CR})$, conversão alimentar (CA), viabilidade $(\mathrm{Vb})$ e fator de produção $(\mathrm{FP})$ de frangos de corte nos períodos acumulados de 1-7, 1-21 e 1-42 dias de idade. (Initial weight (PI), final weight (PF), weight gain (GP), feed intake (CR), feed conversion (CA), viability (Vb) and production factor (FP) for broiler chickens in 1-7, 1-21 and 1-42 days of age).

\begin{tabular}{|c|c|c|c|c|c|c|}
\hline \multirow{2}{*}{$\begin{array}{l}\text { Idade (dias) } \\
\text { Variáveis }\end{array}$} & \multicolumn{3}{|c|}{ Glutamina } & \multicolumn{2}{|c|}{ Nucleotídeos } & \multirow{2}{*}{$\begin{array}{l}\text { CV } \\
(\%)\end{array}$} \\
\hline & 0,0 & 0,5 & 1,0 & 0,0 & 0,04 & \\
\hline \multicolumn{7}{|l|}{$1-7$} \\
\hline Peso inicial, g & 43,5 & 43,4 & 43,4 & 43,5 & 43,4 & 1,38 \\
\hline Peso final, $\mathrm{g}$ & $157^{\mathrm{b}}$ & $161^{\mathrm{ab}}$ & $163^{a}$ & 161 & 159 & 2,93 \\
\hline Ganho de peso médio, g & $112^{b}$ & $117^{\mathrm{ab}}$ & $119^{\mathrm{a}}$ & 117 & 116 & 4,43 \\
\hline Consumo de ração, g & $142^{b}$ & $142^{b}$ & $147^{a}$ & 145 & 143 & 2,32 \\
\hline Conversão alimentar & 1,265 & 1,214 & 1,236 & 1,234 & 1,243 & 4,23 \\
\hline Viabilidade, $\%^{1}$ & 99,50 & 99,50 & 99,50 & 99,64 & 99,33 & 55,30 \\
\hline \multicolumn{7}{|l|}{$1-21$} \\
\hline Peso final, $\mathrm{g}$ & 881 & 878 & 900 & 896 & 878 & 2,42 \\
\hline Ganho de peso médio, g & 838 & 835 & 857 & 852 & 834 & 2,62 \\
\hline Consumo de ração, $\mathrm{g}$ & $1165^{a b}$ & $1159^{b}$ & $1193^{a}$ & 1182 & 1163 & 2,28 \\
\hline Conversão alimentar & 1,406 & 1,399 & 1,413 & 1,405 & 1,407 & 1,38 \\
\hline Viabilidade, $\%^{1}$ & 96,50 & 94,00 & 95,50 & 95,00 & 95,33 & 18,33 \\
\hline \multicolumn{7}{|l|}{$1-42$} \\
\hline Peso final, $\mathrm{g}$ & 2782 & 2799 & 2816 & 2796 & 2802 & 4,21 \\
\hline Ganho de peso médio, g & 2738 & 2756 & 2773 & 2753 & 2759 & 4,27 \\
\hline Consumo de ração, $\mathrm{g}$ & 4751 & 4654 & 4802 & 4751 & 4720 & 2,66 \\
\hline Conversão alimentar & 1,760 & 1,718 & 1,750 & 1,756 & 1,737 & 2,70 \\
\hline Viabilidade, $\%^{1}$ & 95,50 & 99,50 & 97,50 & 93,00 & 92,00 & 14,22 \\
\hline Fator de produção ${ }^{2}$ & 350 & 346 & 347 & 347 & 348 & 5,91 \\
\hline
\end{tabular}

${ }^{1}$ Dados de mortalidade foram submetidos à transformação $(x+0,5) 1 / 2$, antes da Anova. ${ }^{2}$ Fator de produção $=(($ GP $\times$ viabilidade $) / C A) \times 100$. CV: Coeficiente de variação. 
sido adequadamente alojados e as práticas de biosseguridade atendidas, consequentemente, eliminando a possibilidade de desafio para as aves, apesar de estarem sendo criadas no sistema alternativo, dificultando a ação da glutamina e dos nucleotídeos como estimuladores de desempenho.

Estes aditivos poderiam apresentar respostas superiores em condições desfavoráveis de higiene, instalações e desbalanço nutricional. Segundo Menten (2001), é de fundamental a existência de um desafio sanitário de campo suficiente para que os aditivos promotores de crescimento possam produzir efeitos significativos sobre o desempenho de aves e suínos.

Pelícia et al. (2010) não encontraram efeito da inclusão de $0,04 \%$ de nucleotídeos no desempenho de frangos de corte. Por outro lado, Bruno (2009) encontrou efeito positivo no desempenho de frangos de corte utilizando $0,05 \%$ de nucleotídeos na dieta no periodo de 1 a 21 dias de idade.
Nas medidas de altura e área da vilosidade e profundidade de cripta do duodeno, jejuno e íleo dos frangos de corte, não foram observados efeitos da inclusão de glutamina e nucleotídeos nas dietas (tabela III). Também não se verificou efeito sobre proporção de células caliciformes/enterócitos nas três porções do intestino delgado.

O desenvolvimento completo dos vilos do duodeno dos frangos de corte ocorre até os sete dias de idade. Entretanto, o desenvolvimento dos vilos do jejuno e do íleo continua até 14 dias de idade (Uni et al., 1998). Ou seja, a maturação intestinal de frangos de corte ocorre nas duas primeiras semanas de vida, que pode explicar porque não foram observados efeito da glutamina e nucleotídeos na morfologia intestinal aos 42 dias de idade, pois as aves já apresentavam maturação intestinal.

A suplementação de glutamina sugere efeitos positivos na fase de 1 a 7 dias de

Tabela III. Morfometria do intestino delgado de frangos de corte aos 42 dias de idade. (Intestinal morphometry of 42 days old broiler chickens).

\begin{tabular}{|c|c|c|c|c|c|c|}
\hline \multirow[b]{2}{*}{ Variáveis } & \multicolumn{3}{|c|}{ Glutamina } & \multicolumn{2}{|c|}{ Nucleotídeos } & \multirow{2}{*}{$\begin{array}{l}\text { CV } \\
(\%)\end{array}$} \\
\hline & 0,0 & 0,5 & 1,0 & 0,0 & 0,04 & \\
\hline \multicolumn{7}{|l|}{ Duodeno } \\
\hline Altura, $\mu \mathrm{m}$ & 1177 & 1220 & 1086 & 1184 & 1138 & 19,96 \\
\hline Área, $\mu \mathrm{m}$ & 173942 & 149268 & 158389 & 174687 & 146380 & 38,31 \\
\hline Prof. cripta, $\mu \mathrm{m}$ & 118 & 120 & 115 & 122 & 113 & 16,26 \\
\hline Caliciforme/enterocítos ${ }^{1}$ & 52,92 & 49,65 & 54,30 & 50,18 & 53,73 & 14,36 \\
\hline \multicolumn{7}{|l|}{ Jejuno } \\
\hline Altura, $\mu \mathrm{m}$ & 1167 & 1081 & 955 & 1096 & 1106 & 13,98 \\
\hline Área, $\mu \mathrm{m}$ & 136621 & 109671 & 110307 & 120502 & 117230 & 28,43 \\
\hline Prof. cripta, $\mu \mathrm{m}$ & 114,54 & 117,02 & 107,06 & 117,95 & 107,80 & 15,87 \\
\hline Caliciforme/ enterocítos ${ }^{1}$ & 52,97 & 54,20 & 55,65 & 54,07 & 54,46 & 12,00 \\
\hline \multicolumn{7}{|l|}{ íleo } \\
\hline Altura, $\mu \mathrm{m}$ & 649 & 660 & 643 & 709 & 593 & 19,41 \\
\hline Área, $\mu \mathrm{m}$ & 70198 & 64734 & 80887 & 77356 & 66523 & 39,51 \\
\hline Prof. cripta, $\mu \mathrm{m}$ & 96,09 & 106,94 & 95,37 & 101,48 & 97,46 & 22,43 \\
\hline Caliciforme/enterocítos ${ }^{1}$ & 55,55 & 56,85 & 57,98 & 55,19 & 58,39 & 8,38 \\
\hline
\end{tabular}

${ }^{1}$ Porcentagem de células caliciformes em relação aos enterócitos (obtida pela contagem de células caliciformes a cada 500 enterócitos). CV: Coeficiente de variação. 
Tabela IV. Peso relativo dos órgãos ${ }^{1}$ (\%) e comprimento de intestinos (cm) aos 42 dias de idade. (Relative organs weigth (\%) and size of intestine $(\mathrm{cm})$ of 42 days old broiler chickens).

\begin{tabular}{|c|c|c|c|c|c|c|}
\hline \multirow[b]{2}{*}{ Variáveis } & \multicolumn{3}{|c|}{ Glutamina } & \multicolumn{2}{|c|}{ Nucleotídeos } & \multirow{2}{*}{$\begin{array}{l}\text { CV } \\
(\%)\end{array}$} \\
\hline & 0,0 & 0,5 & 1,0 & 0,0 & 0,04 & \\
\hline Moela, \% & 1,24 & 1,25 & 1,29 & 1,25 & 1,27 & 12,66 \\
\hline Proventrículo, \% & 0,39 & 0,34 & 0,35 & 0,31 & 0,38 & 18,21 \\
\hline Fígado, \% & 1,78 & 1,74 & 1,72 & 1,74 & 1,76 & 10,41 \\
\hline Pâncreas, \% & 0,17 & 0,18 & 0,18 & 0,18 & 0,17 & 12,08 \\
\hline Intestino delgado, \% & 2,96 & 2,78 & 2,82 & 2,83 & 2,87 & 13,70 \\
\hline Intestino grosso, \% & 0,59 & 0,67 & 0,65 & 0,65 & 0,62 & 22,83 \\
\hline Intestino delgado, $\mathrm{cm}$ & 146,37 & 140,50 & 144,00 & 140,67 & 146,58 & 7,35 \\
\hline Intestino grosso, cm & 40,00 & 41,38 & 41,75 & 40,08 & 42,00 & 9,29 \\
\hline
\end{tabular}

${ }^{1}$ Peso relativo de órgãos $(\%)=$ (peso do órgão, g/peso vivo, g) x 100. CV: Coeficiente de variação.

idade sobre a maturação intestinal, que envolve mudanças morfológicas e fisiológicas, proporcionado aumento na área de superfície de digestão e de absorção, melhorando o desempenho durante o período inicial de desenvolvimento do trato digestivo. Esta melhora na estrutura da mucosa intestinal foi demonstrada em frangos de corte por Maiorka et al. (2002) e Sakamoto et al. (2005), nos quais, trabalhando com glutamina na primeira semana de idade, observaram melhor desenvolvimento da mucosa intestinal, mostrando que esse aminoácido tem papel importante na maturação do intestino dos pintos, que ocorre nos primeiros dias de vida das aves.

Os pesos relativos dos órgãos e comprimento dos intestinos não foram influenciados pela inclusão de glutamina e nucleotídeos na dieta (tabela IV). Segundo Rao e McCracken (1992) os pesos dos órgãos variam com o consumo de energia e/ ou proteína, sugerindo que mantidas as mesmas quantidades, os pesos serão semelhantes.

De acordo com a literatura, a demanda por produtos da avicultura alternativa, face às exigências de mercado e às proibições do uso de alguns antibióticos promotores de crescimentos, tem provocado melhoria das técnicas de criação e dos resultados zootécnicos (Butolo, 2003). Portanto, a suplementação de glutamina pode ser uma alternativa, pois tem ação trófica na mucosa intestinal e no sistema imune dos animais, melhorando o desempenho (Maiorka et al., 2002).

\section{CONCLUSÕES}

A suplementação de glutamina e nucleotídeos em dietas de frangos de corte criados no sistema alternativo não influencia o desempenho e a morfologia intestenial. Pode-se utilizar $1,0 \%$ de inclusão de glutamina no período de 1 a 21 dias de idade, pois favorece o desempenho das aves.

\section{BIBLIOGRAFIA}

Bruno, J.B.C. 2009. Efeito dos diferentes níveis de nucleotídeos em frangos de corte alimentados com probióticos (Dissertação). Universidade de São Paulo. Pirassununga, SP.

Butolo, J.E. 2003. Produção de frangos alternativos. Simpósio sobre Nutrição de Aves e Suínos. Anais... CBNA. Cascavel. pp: 75-82.

Calson, M.S., Veum, T.L. and Turk, J.R. 2005.

Archivos de zootecnia vol. 60, núm. 232, p. 919. 


\section{ZAVARIZE, SARTORI, PELÍCIA, PEZZATO, ARAUJO, STRADIOTTI E MADEIRA}

Effects of yeast extract versus animal plasma in weanling pig diets on growth performance and intestinal morphology. J. Swine Health Prod., 13: 204-209.

Dell'Orto, V., Di Giancamillo, A. and Savoini, G. 2002. Influence of nucleotides and glutamine dietary supplementation on gut health of weanling piglets. J. Anim. Sci., 80: 220

Demattê Filho, L.C. e Mendes, C.M.I. 2001. Viabilidade técnica e econômica na criação alternativa de frangos. In: Conferência Apinco de Ciência e Tecnologia Avícolas. Anais... Facta. Campinas. pp. 55-266.

Dewitt, R.C., Wu, Y.E and Renegar, K. 1999. Glutamine-enriched total parental nutrition preserves respiratory immunity and improves survival to a Pseudomonas pneumonia. J. Surg. Res., 84: 13-18.

Lobley, G.E., Hoskin, S.O. and McNeil, C.J. 2001. Glutamine in animal science and production. J. Nutr., 131: 2525S-2531S.

Maiorka, A., Boleli, I.C. e Macari, M. 2002. Desenvolvimento e reparo da mucosa intestinal. In: Macari, M., Furlan, R.L., Gonzales, E. Fisiologia aviária aplicada a frangos de corte. FUNEP/UNESP. Jaboticabal. pp. 113-123.

Menten, J.F.M. 2001. Aditivos alternativos na produção de aves: probióticos e prebióticos. In: Reunião Anual da Sociedade Brasileira de Zootecnia, 38. Anais... SBZ. Piracicaba. pp.141-157.

Pelícia, V.C., Sartori, J.R., Zavarize, K.C., Pezzato, A.C., Stradiotti, A.C., Araujo, P.C., Mituo, M.A.O. e Madeira, L.A. 2010. Effect of nucleotides on broiler performance and carcass yield. Rev. Bras. Ciênc. Avíc., 12: 31-34.

Pierzynowski, S.G., Pirdra, V.J.L., Hommel-Hansen, T. and Studzinski, T. 2001. Glutamine in gut metabolism. In: Piva, A., Knudsen, K.E.B., Lindberg, J.E. Gut environment of pigs. University Press. Nottingham. pp. 43-62.
Rao, D.S. and Mccracken, K.J. 1992. Energy: protein interactions in growing boars on hegh genetic potencial for lean growth: I Effects on growth, carcass characteristics and organ weights. Anim. Prod., 54: 75-82.

Rostagno, H.S., Albino, L.F.T., Donzele, J.L., Gomes, P.C., Oliveira, R.F., Lopes, D.C., Ferreira, A.S. e Barreto, S.L.T. 2005. Tabelas brasileiras para aves e suínos: composição de alimentos e exigências nutricionais. UFV. Viçosa. pp.186.

Sakamoto, M.I. 2005. Influência da glutamina e vitamina $E$ sobre o desempenho, resposta imunológica e morfometria intestinal de frangos de corte. (Dissertação de Mestrado). Universidade Estadual de Maringá. Maringá, PR.

SAS. 1996. SAS/STAT. User's guide. Version 6.11. $4^{\mathrm{a}}$ ed. V. 2. SAS Institute Inc. Cary. 842 pp.

Tucci, F.M. 2003. Efeito da adição de agentes tróficos na dieta de leitões desmamados sobre a renovação celular da mucosa intestinal, enzimas digestivas e desempenho. (Tese de doutorado). Faculdade de Ciências Agrárias e Veterinárias. Universidade Estadual Paulista. Jaboticabal, SP. 85 pp.

Uauy, R., Quan, R. and Gil, A. 1994. Role of nucleotides in intestinal development and repair: implications for infant nutrition. J. Nutr., 124: 1436S-1441S.

Uni, Z., Ganot, S.E. and Sklan, D. 1998. Post-hatch development of mucosal function in the broiler small intestine. Poult. Sci., 77: 75-82.

Yu, I.T., Wu, J.F., Yang, P.C., Liu, C.Y., Lee, D.N. and Yen, H.T. 2002. Role of glutamine and nucleotides in combination in growth, immune responses and FMD antibody titers of weaned pigs. Anim. Sci., 75: 379-385.

Wu, G., Meier, S.B. and Knabe, D.A. 1996. Dietary glutamine supplementation prevents jejunal atrophy in weaned pigs. J. Nutr., 126: 25782584.

Archivos de zootecnia vol. 60, núm. 232, p. 920. 